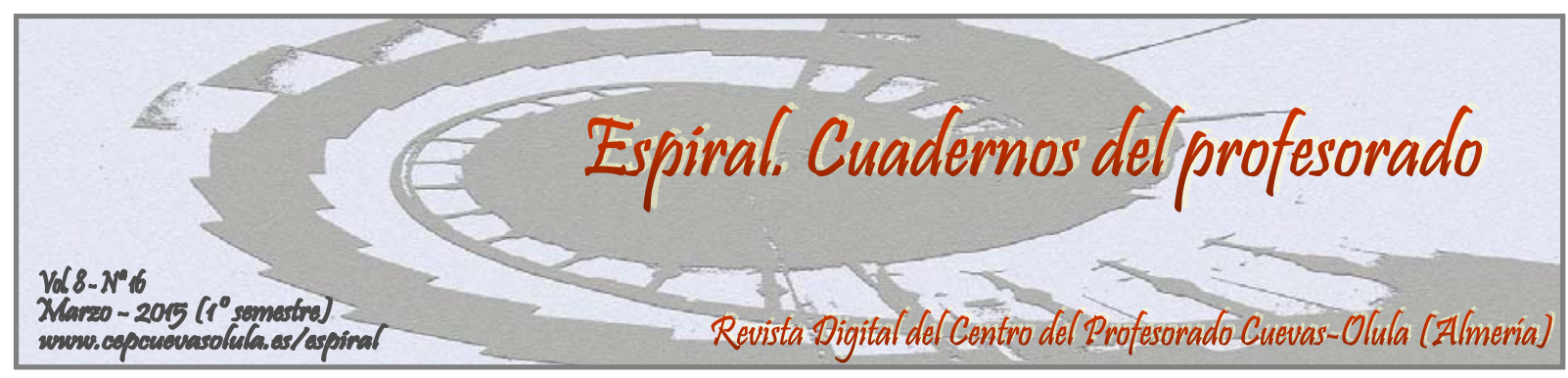

\title{
DEL AULA A LA BITÁCORA. APRENDIENDO A APRENDER
}

FROM THE CLASSROOM TO THE BINACLE. LEARNING TO LEARN

\section{Mariano García Fernández}

IES Jaroso de Cuevas del Almanzora, Almería, España

RESUMEN: Este trabajo explica cómo los principios de la inteligencia emocional pueden repercutir de manera positiva en las herramientas de evaluación y por ende, en la superación del módulo de Empresa e Iniciativa Emprendedora en $2^{\circ}$ curso del ciclo de grado medio de Soldadura y Calderería. Como metodología, se ha utilizado el estudio de casos y como técnica de investigación la triangulación de datos; a partir de la entrevista personal, el cuestionario y la observación directa. Asimismo, esta triangulación de datos realizada de forma cualitativa se ha completado con un estudio cuantitativo a partir de las medianas de los cuestionarios. La recogida de datos se ha realizado mediante la bitácora http://economia-bach.blogspot.com/. Los resultados obtenidos explican que los principios de la inteligencia emocional, pueden ayudar al alumnado a adquirir competencias en la búsqueda de herramientas para la mejora continua. Por otro lado, se puede observar que la utilización de la inteligencia emocional por parte del alumnado, y guiada por el docente, puede derivar en mejoras significativas, en el cumplimiento de los objetivos, para una mayor eficiencia y mejora del sistema evaluación.

Palabras clave: Inteligencia emocional, Evaluación, Empresa, Iniciativa Emprendedora, TIC.

ABSTRACT: This paper explains how the principles of emotional intelligence can impact, positively assessment tools and thus overcoming the module in Enterprise and Entrepreneurship 2nd year in middle grade cycle Welding and Boiler. As a methodology, we have used the case study as a research technique and the triangulation of data, from the personal interview, questionnaire and direct observation. Also, this data triangulation qualitatively performed and completed with a quantitative survey of the medium from the questionnaires. Data collection was performed using the log http://economia-bach.blogspot.com/. The results explain that the principles of emotional intelligence can help students acquire skills in seeking continuous improvement tools. On the other hand, it can be seen that the use of emotional intelligence on the part of students and guided by the teacher, can lead to significant improvements in the performance of objectives for efficiency and improvement of evaluation system.

Key words: emotional intelligence, evaluation, business, entrepreneurship, TIC.

García Fernández, M. (2015). Del aula a la bitácora. Aprendiendo a aprender. Espiral. Cuadernos del Profesorado, 8(16), 50-55. Disponible en: http://www.cepcuevasolula.es/espiral.

Fecha de recepción: 19/06/2013

Fecha de aceptación: 20/03/2015
Enviar correspondencia a: mariano.garcía.edu@juntadeandalucia.es 


\section{1.- INTRODUCCIÓN}

Ante los diferentes cambios de hoy en día: globalización, TIC, etc., los individuos y por ende, las organizaciones, tienen que optar por diferentes cambios. Uno de los principales cambios es la adaptación a las nuevas situaciones. La adaptación no siempre se puede realizar de manera óptima, ya que pueden faltar habilidades o aptitudes, pero podemos disminuir el gap existente, a través de la inteligencia. Ahora bien, existe un nuevo problema, qué tipo de inteligencia utilizar: inteligencia en aptitudes para poder observar y repetir elementos y variables, inteligencia relacionada con el cociente intelectual, inteligencia abstracta... El hecho es que no es necesario tener un cociente intelectual muy alto para poder adaptarse a los cambios pero sí hace falta lo que se podría tipificar como inteligencia emocional. Ésta se ocupa, principalmente, de la adaptación del individuo ante diferentes situaciones.

A partir de lo anterior, en la sociedad encontramos diversos ejemplos de ello, un alumno sin ser muy brillante en sus estudios, y a partir de su inteligencia emocional, puede obtener un puesto con un mayor status en una empresa. O por otro lado, un emprendedor/a puede tener un mayor éxito teniendo un cociente intelectual menor que otros emprendedores/as. Algún arduo lector, puede pensar que estos ejemplos también pueden tener como causa otros aspectos significativos, como por ejemplo, una mayor inversión o "estar en el momento preciso", pero no negarán que son muchos los factores que intervienen y que cabe la posibilidad de que entre uno de ellos esté la inteligencia emocional.

Por otro lado, se observa la dualidad del alumno entre aprender y/o superar una asignatura o módulo profesional. Muchos de los alumnos intentan aprender y de este modo superar sus materias y otro alumnado únicamente superar éstas. En este sentido, el docente ha de intentar guiar al alumno por ambos caminos, ya que en muchas ocasiones y en el mejor de los casos el alumnado aprueba por repetición y acotación. El segundo escollo es la evaluación, donde encontramos alumnos dispuestos a aprender y superar los módulos, pero tienen deficiencias en interpretar y/o adaptarse al sistema de evaluación propuesto por el docente. Una manera de superar este problema es mediante el uso y la potenciación de la inteligencia emocional. De ahí la importancia del estudio. En este sentido, el profesorado ha de adaptarse al momento que le ha tocado vivir. Esto supone no solo un cambio metodológico sino nueva relación entre el alumno y el profesor. No será esta una experiencia puntual, es un modo de trabajo. Se utilizan las potencialidades de las TIC como manera de abordar los objetivos curriculares del módulo Empresa e Iniciativa emprendedora consiguiendo la mejora de la motivación de los alumnos, su forma de participar en clase y la mejora de los resultados académicos, en definitiva de todo el proceso de enseñanza aprendizaje.

Los objetivos principales de la investigación son observar si la inteligencia emocional tiene una influencia positiva en la evaluación de las enseñanzas y qué características de la inteligencia emocional son las que en mayor medida inciden en el éxito de la evaluación, en caso de obtener una relación positiva. Así mismo, si la inteligencia emocional es una buena herramienta, entre el profesorado y el alumnado, para el éxito en un sistema evaluación.

La investigación se ha estructurado en primer lugar, por medio de una revisión teórica sobre la inteligencia emocional y sobre la relación que tiene ésta con la evaluación, Seguidamente se ha tratado la metodología del estudio. En el tercer bloque de contenidos, se han planteado las conclusiones, limitaciones y futuros estudios.

\section{2.- INTELIGENCIA EMOCIONAL}

La inteligencia emocional es el uso inteligente de las emociones (Weisinger, 1998). Asimismo, Gardner (1993), define inteligencia emocional como "El potencial biopsicológico para procesar información que puede generarse en el contexto cultural para resolver los problemas". Por otro lado, la inteligencia emocional se convierte en una habilidad para procesar la información emocional que incluye la percepción, la asimilación, la comprensión y la dirección de las emociones (Mayer y Cobb, 2000). 


\section{Principios}

La siguiente cuestión, una vez definida lo que es inteligencia emocional, se han expuesto los principios básicos en los que se basa la inteligencia emocional. En este sentido la inteligencia emocional, tiene los siguientes principios (Gómez, Galiana, y León, 2000):

- Autoconocimiento. Capacidad para conocerse uno mismo y saber los puntos fuertes y débiles.

- Autocontrol. Capacidad para controlar los impulsos, saber mantener la calma y no perder los nervios.

- Automotivación. Habilidad para realizar cosas por uno mismo.

- Empatía. Intentar comprender la situación del otro.

- Habilidades sociales. Capacidad para relacionarse con otras personas, ejercitando dotes comunicativas para lograr un acercamiento eficaz.

- Asertividad. Saber defender las propias ideas no respetando la de los demás.

- Proactividad. Habilidad para tomar la iniciativa ante oportunidades o problemas, responsabilizándose de sus propios actos.

- Creatividad. Competencia para observar el mundo desde otra perspectiva.

Este conjunto de principios expuestos, son requisitos para que se dé la inteligencia emocional. Así mismo, para medir la inteligencia emocional en la sociedad, la literatura ha llevado a cabo diversos modelos de inteligencia emocional desde diferentes perspectivas.

\section{Principales modelos de Inteligencia emocional} modelos.

La inteligencia emocional distingue entre diferentes modelos: mixtos, de habilidades y otros

Con relación a los modelos mixtos, los modelos que en mayor medida son más reconocidos por la literatura son los de Goleman y Bar-On. Éstos incluyen rasgos de personalidad como el control del impulso, la motivación, la tolerancia a la frustración, el manejo del estrés, la ansiedad, la asertividad, la confianza y/o la persistencia.

Con relación a los modelos de habilidades, son los que fundamentan el constructo de inteligencia emocional en habilidades para el procesamiento de la información emocional. En este sentido, estos modelos no incluyen componentes de factores de personalidad, siendo el más relevante de estos modelos el de Salovey y Mayer. Éstos postulan la existencia de una serie de habilidades cognitivas o destrezas de los lóbulos prefontales del neocórtex para percibir, evaluar, expresar, manejar y autorregular las emociones de un modo inteligente y adaptado al logro del bienestar, a partir de las normas sociales y los valores éticos.

Con relación a otros modelos, se puede observar que Incluyen componentes de personalidad, habilidades cognitivas y otros factores de aportaciones de personales, que en algunos casos son fruto de constructos creados ad hoc con la finalidad de enfatizar el sentido popular y divulgativo del constructo inteligencia emocional. Entre los más representativos están los modelos de: Matineaud y Engelharth (1996), Cooper y Sawaf (1997), Rovira (1998), Elías, Tobías y Friedlander (1999), Higgins, Grant y Shah (1999), Vallés y Vallés (1999), Barret y Gross (2001) y Bonano (2001).

Una vez estudiados los principales modelos sobre inteligencia emocional, se observa qué relación tiene ésta con el rendimiento a través de la evaluación de las enseñanzas.

\section{Inteligencia emocional y evaluación}

Numerosos autores han estudiado la relación existente entre inteligencia emocional y evaluación. Por ejemplo, Sternberg (1998) señala que cuesta mucho admitir que un individuo sea muy habilidoso para resolver problemas lógicos y/o matemáticos, sin embargo no lo sea tanto para otro tipo de problemas. Para ello, Schutte et al. (1996) realizaron un instrumento de medida para predecir el éxito de académico de los estudiantes. Era una escala basada en Salovey y Mayer (1990). Los 
resultados de este estudio predecían de forma directa el nivel de éxito directo aunque sólo durante el primer año. Más tarde (2001), la Universidad de Málaga analizó este constructo pero utilizando un mediador como la salud mental. Estas investigaciones obtuvieron relaciones satisfactorias sobre la relación entre inteligencia emocional y evaluación positiva.

\section{3.- MÉTODO}

\section{Muestra}

Para el correcto desarrollo de la metodología, se ha utilizado el método del caso utilizando evidencias cualitativas y cuantitativas de los alumnos de $2^{\circ}$ del ciclo de grado medio de Soldadura y Calderería, curso 2012-2013.

Los alumnos seleccionados fueron doce que correspondían a su totalidad, con lo cual no hacía falta realizar un muestreo, ya que el universo de estudio era el 100\% de los alumnos que asistían a clase regularmente. Para el correcto desarrollo del estudio, se realizó un protocolo. Éste se basó en las siguientes etapas llevadas a cabo en el siguiente orden: objetivos, criterios de selección de los alumnos, fuentes de recogida de datos, acceso a los alumnos, caso piloto y resto de casos, presentación de informes y resultados, planteamiento de conclusiones y límites al estudio.

\section{Instrumentos}

Se realizó una triangulación de datos para obtener unos resultados óptimos: entrevistas personales, cuestionario y la observación directa. Dicha triangulación se basó en las bases conceptuales de la perspectiva Escuela 2.0, realizándose una bitácora http://economiabach.blogspot.com/, donde se introdujo la entrevista para que previamente la tuvieran los alumnos. Asimismo, se cumplimentó el cuestionario por este medio.

Las entrevistas personales, se realizaron con cada uno de los alumnos individualmente, en horas no lectivas. Las entrevistas personales fueron grabadas, con permiso previo por parte de los alumnos, todos mayores de edad, para posteriormente volver a oírlas y completar la información obtenida in situ. Cada una de las entrevistas personales, doce en total, consistió en una entrevista personal con una serie de preguntas abiertas. Asimismo, se realizó un cuestionario basado en preguntas cerradas que permitió ampliar y depurar la información obtenida de las entrevistas basadas en preguntas abiertas.

Con relación a la observación directa (participante), se realizó a través del trato diario en el aula.

Con relación a las cuestiones tratadas en las entrevistas con preguntas abiertas, se analizaron Los principios de la inteligencia emocional y los instrumentos de evaluación en el módulo de Empresa e Iniciativa Emprendedora, anteriormente expuestos.

De cada dimensión, se realizaron una serie de preguntas abiertas para analizar el grado de desarrollo de la dimensión analizada en los alumnos objeto de estudio. En concreto, se utilizaron 28 preguntas abiertas para evaluar los dos constructos inteligencia emocional y sistema de evaluación y sus diferentes dimensiones; siete en el caso de principios de la inteligencia emocional y tres en el caso de instrumentos de evaluación. Con relación a las cuestiones analizadas a través del cuestionario, se han medido las dimensiones de la inteligencia emocional y del sistema evaluación utilizando 30 ítems en una escala de 7 puntos (de 1 totalmente en desacuerdo a 7 totalmente de acuerdo).

\section{Recogida de datos}

Una vez expuestos los objetivos y seleccionados los alumnos, y siguiendo un protocolo de actuación, se pasó a la recogida de datos. Con relación a la recogida de datos, tanto primarios como secundarios, el estudio utiliza tres fuentes de datos: observación directa, cuestionario y entrevista personal.

Una vez definidos las fuentes de recogidas de datos y mediante un sorteo, se realizó un caso piloto con un alumno seleccionado al azar para observar errores si los instrumentos eran entendidos 
por el alumno. Tras las correcciones y rectificaciones en la entrevista personal y el cuestionario, éstas se realizaron al resto de casos. Finalmente, se realizaron los informes de resultados.

\section{Análisis de datos}

Con toda la información recabada, se utilizó, como técnica de análisis, la triangulación de datos. Ésta proporciona validez (del constructo, interna y externa) y fiabilidad de los resultados (Yin, 1994). Con relación a la validez del constructo, se ha realizado un marco teórico. En este sentido, para conseguir la validez interna, se han utilizado patrones de causalidad y la construcción de explicaciones, a través de la descripción de interacciones múltiples y el seguimiento de los casos. Asimismo, se incrementó la validez externa por medio de la réplica de la investigación en los diferentes casos y la comparación de resultados entre éstas. Por otro lado, con relación a la fiabilidad, se diseñó y aplicó un protocolo de estudio de caso detallado tal como se ha comentado anteriormente.

\section{4.- RESULTADOS Y CONCLUSIONES}

El estudio muestra que existe una relación positiva entre inteligencia emocional y éxito en el sistema de evaluación, esto es, los alumnos que en mayor medida utilizan iniciativas basándose en la inteligencia emocional obtienen mayores resultados. Entre las características principales que utiliza el alumnado que obtiene mayor éxito se encuentran: automotivación, tolerar la autofrustación, mostrar optimismo, ser un buen conocedor del comportamiento de los demás, sonreír, aprender de los errores, controlar los miedos y conocer los defectos personales y la necesidad de cambiar. Así mismo, entre las características que en menor medida influyeron se sitúan: calmar a los demás, ser capaz de tranquilizarse, ser realista y valorar opiniones.

Por otro lado, el estudio ha determinado que la inteligencia emocional puede ser una buena herramienta para el profesorado. En este sentido, puede ser útil, tanto dentro del aula como fuera de ésta. Dentro del aula porque puede hacer servir los diferentes modelos sobre el constructo inteligencia emocional, de manera dinámica con el fin de obtener por ejemplo, un sistema de evaluación acorde a la inteligencia emocional del alumnado, siempre y cuando se haya medido ésta. Por otro lado, también es útil fuera del aula porque se puede adaptar y/o realizar, tanto la programación didáctica como la programación de aula, de manera sinérgica con la inteligencia emocional del alumnado. De este modo, se puede solventar el gap que existe entre profesores y alumnos, a la hora de demandar y ofrecer como comunidad asociada, de manera inseparable.

La inteligencia emocional del alumnado puede guiarles para mejorar sus rendimientos académicos. En este sentido, la convergencia entre inteligencia emocional-evaluación puede ser productiva para el alumnado. En el presente estudio, los alumnos que de alguna manera utilizaron o aprendieron utilizando, en mayor medida características de la inteligencia emocional, obtuvieron mejores resultados.

\section{Limitaciones y futuras líneas de investigación}

La principal limitación que tiene es estudio es que no existen muchas evidencias empíricas con las que se puedan contrastar y comparar. No obstante en futuras investigaciones, y para solventar esta limitación, se va a proceder a su medición psicosométrica.

Otra limitación que tiene el estudio es que no existen evidencias empíricas con las cuales contrastarlo y compararlo. Asímismo, los resultados no pueden extrapolarse a otras clases o a otros alumnos, ya que la metodología utilizada no exige una muestra significativa. No obstante, en futuras investigaciones, y para solventar estas limitaciones, se va a proceder a un análisis empírico en una muestra más amplia.

\section{5.- REFERENCIAS}

Bar-On, R. (1988). The development of a concept of psycholoogical well-being, Unpublished doctoral dissertation, Rodhes University, South Africa. 
Bar-On, R. (1997). The Emotional Quotient inventory (EQ-I): Techical Manual, Toronto, Canadá: Multi-Health Systems.

Barret, L. F. y Gross, J. J. (2001). Emotional Intelligence. A process model of emotion representation and regulation. En T. J. Mayne y G. A. Bonano (Eds.). Emotions, Current Issues and future directions, New York: The Guilford Press.

Bonano, G. A. (2001). Emotion self-regulation. En T.J. Mayne y G.A. Bonano (Eds.), Emotions, Current sigues and future directions, New Cork: The Guildford Press.

Cooper, R. K. y Sawaf, A. (1997). Estrategia emocional para ejecutivos, Barcelona: Martínez Roca.

Elias, M., Tobias, S., y Friedlander, B. (1999). Educar con inteligencia emocional, Barcelona: Plaza y Janés.

Gardner, H. (1993). Multiples Intelligences, New York: BasicBooks, Traducción española en Ed. Paidós.

Goleman, D. (1995). Emotional intelligence, New York: Bantam.

Goleman, D. (1995). What's your emotional intelligence quotient? You'll soon find out. Utne Reader, November/December.

Goleman, D. (1998). “What makes a leader?”, Harvard Business Review, November-December.

Goleman, D. (1998). Working with emotional intelligence, Nw York: Bantman.

Gómez Gras, J. M., Galiana Lapera, D., y León Espí, D. (2000): Que debes saber para mejorar tu empleabilidad, Universidad Miguel Hernández de Elche.

Higgins, E. T., Grant, H., y Shah, J. (1992). Seft Regulation and quality of life: Emotional and non-emotional life experiences. En Kahneman, Diener y Sxhwarz (Eds.), Well-being: the foundations of hedonic psychology, New York: Rusell Sage Foundation.

Martineaud, S. y Engelhart, D. (1996). El Test de inteligencia emocional, Barcelona: Martínez Roca.

Mayer, J. D. y Cobb, C. D. (2000). Educational policy on emocional intelligence: the case for ability scales. In R. Bar-On \& J. D. A. Parker (Eds.), The handbook of emotional intelligence, San Francisco, Jossey Bass.

Mayer, J. D. y Cobb, C. D. (2000). Educational policy on emocional intelligence: the case for ability scales. In R. Bar-On \& J. D. A. Parker (Eds.), The handbook of emotional intelligence, San Francisco, Jossey Bass.

Mayer, J. D., Salovey, P., y Caruso, D.R. (2000). Models of emotional intelligence, In R. J. Sternberg (Ed.), Handbook of intelligence (pp.3 96-420). Cambridge, England: Cambridge University Press.

Rovira (1998). Cómo saber si un es emocionalmente inteligente. Aloma, 2, 57-68.

Schutte, N. S., Malouff, J. M., Hall, L. E., Haggerty, D. J., Cooper, J. T., Golden, C. J., y Dornheim, L. (1998). Development and validation of a measure of emotional intelligence. International Journal of Research into Structure and Development of Personality, and the Causation of Individual Differences, Vol. 25, N. 2, pp. 167-178.

Sternberg, R. J. (1996). Successful intelligence. How practical and creative intelligence determine success in life. Hillsdale, N.J.: Simon y Schuster.

Vallés, A. y Vallés, C. (1999). Desarrollando la inteligencia emocional, Madrid: EOS.

Weisinger, H. (1988): Emocional intelligence at work: The untapped edge for succes, Josey-Bass, San Francisco.

Yin R. K. (1994). Case study research: design and methods. Sage, Thousand Oaks.

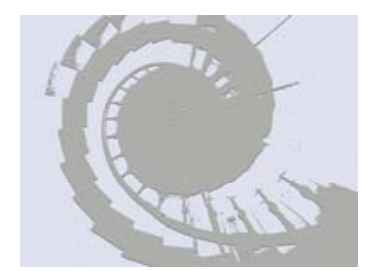

